Voix et Images

voixetimages

\title{
Bibliographie de France Daigle
}

\section{Benoit Doyon-Gosselin}

Volume 29, numéro 3 (87), printemps 2004

France Daigle

URI : https://id.erudit.org/iderudit/009224ar

DOI : https://doi.org/10.7202/009224ar

Aller au sommaire du numéro

Éditeur(s)

Université du Québec à Montréal

ISSN

0318-9201 (imprimé)

1705-933X (numérique)

Découvrir la revue

Citer ce document

Doyon-Gosselin, B. (2004). Bibliographie de France Daigle. Voix et Images, 29(3),

101-107. https://doi.org/10.7202/009224ar d'utilisation que vous pouvez consulter en ligne.

https://apropos.erudit.org/fr/usagers/politique-dutilisation/ 


\section{B I B L I O G R P H I E D F R A C E D A I G L E}

$++$

\section{BENOIT DOYON-GOSSELIN}

Université de Moncton

\section{E U V R E S}

I. 1 . Ro m a n s

+ Sans jamais parler du vent. Roman de crainte et d'espoir que la mort arrive à temps, Moncton, Éditions d'Acadie, 1983, $141 \mathrm{p}$.

+ Film d'amour et de dépendance. Chef-d'œuvre obscur, Moncton, Éditions d'Acadie, 1984, 119 p.

+ Histoire de la maison qui brûle. Vaguement suivi d'un dernier regard sur la maison qui brûle, Moncton, Éditions d'Acadie, 1985, $107 \mathrm{p}$.

+ Variations en B et K. Plans, devis et contrat pour l'infrastructure d'un pont, Outremont, Éditions nbj, 1985, $44 \mathrm{p}$.

+ L'été avant la mort (en collaboration avec Hélène Harbec), Montréal, Éditions du remue-ménage, 1986, 77 p.

+ La beauté de l'affaire. Fiction autobiographique à plusieurs voix sur son rapport tortueux au langage, Outremont/Moncton, Éditions nbj/Éditions d'Acadie, 1991, 54 p.

+ La vraie vie, Montréal/Moncton, l'Hexagone/Éditions d'Acadie, 1993, 71 p.

+ 1953. Chronique d'une naissance annoncée, Moncton, Éditions d'Acadie, 1995, 165 p.

+ Pas pire, Moncton, Éditions d'Acadie, 1998, 169 p.

+ Pas pire, 2e éd., Montréal, Boréal, coll. «Boréal compact», 2002, 210 p.

+ Un fin passage, Montréal, Boréal, 2001, 130 p.

+ Petites difficultés d'existence, Montréal, Boréal, 2002, 189 p.

\section{2. Poèmes et textes publiés dans des périodiques}

+ «Poème impossible à finir», Éloizes, no 4, automne 1981, p. 15.

+ «Sur les traces de Marianne Godbout, cordonnière et savetière», Éloizes, nº 4, automne 1981, p. 51-53.

+ «Le taillant de la lame», Éloizes, nº 5, printemps 1982, p. 23.

+ «Méditerranéennes 1», Éloizes, n 7, printemps 1983, p. 75.

+ «Méditerranéennes 2», Éloizes, n 7, printemps 1983, p. 76.

+ «Brumes anesthétiques», Éloizes, n 7, printemps 1983, p. 77.

+ «Le pays chaud», Éloizes, n 8, automne 1983, p. 14.

+ «Pensée simple», Éloizes, n 8, automne 1983, p. 14.

+ «Pensée composée», Éloizes, n 8, automne 1983, p. 14.

+ «Pour Zahava où qu'elle soit», Éloizes, n 9, printemps 1984, p. 43.

+ «Et cela dura», Éloizes, no 11, printemps 1985, p. 16-17.

+ «Insulaires. Chronique d'un pays blanc», Estuaire, nº 36, été 1985, p. 61-78. 
+ «Journal pour un être cher», Cahiers bleus, nos 36-37, printemps 1986, p. 13-15.

+ «Sa chienne Chloé», Éloizes, n 16, printemps 1991, p. 83.

+ «Son état ce jour-là», Éloizes, n 16, printemps 1991, p. 84.

+ «Souvenir de voyage», Éloizes, n 16, printemps 1991, p. 85-86.

+ «Son côté scorpion», Éloizes, n 16 , printemps 1991, p. 87-89.

+ «Le concert», Éloizes, n 19, printemps 1993, p. 11.

+ «La biscotte italienne», Éloizes, n 19, printemps 1993, p. 12.

+ «La romance du vin», Éloizes, no 19, printemps 1993, p. 13.

+ «Performance sur Albinoni», Éloizes, n 19, printemps 1993, p. 14.

+ «Le principe de la culture», Éloizes, n 19, printemps 1993, p. 15.

+ «Série noire», Éloizes, n 19, printemps 1993, p. 16-17.

+ «Què'que chose de nouveau», Éloizes, nos 20-21, printemps 1994, p. 119-122.

+ «Étude \# 27», Estuaire, n 78, août 1995, p. 25-27.

+ «Pas pire [extrait]», Europe, no 853, mai 2000, p. 215-219.

+ «Tending Towards the Horizontal: Text», Tessera, n 13, 2002, p. 63-73.

+ «Une petite place», Dalhousie French Studies, n 62, printemps 2003, p. 9.

+ «Cherchez l'imposture», Dalhousie French Studies, n 62, printemps 2003, p. 10.

+ «Simulacre», Dalhousie French Studies, n 62, printemps 2003, p. 11.

\section{I.3. Articles publiés dans des périodiques}

+ «Autoportrait», Québec français, décembre 1985, p. 40.

+ «En me rapprochant sans cesse du texte», La Nouvelle Barre du jour, n 182, septembre 1986, p. 31-45.

\section{4. Publications dans des anthologies ou ouvrages collectifs}

+ «Écriture et américanité» et «Manifeste pour un camping bien articulé», Les cent lignes de notre américanité. Actes de colloque tenu à Moncton du 14 au 16 juin 1984, Moncton, Éditions Perce-Neige, 1984, p. 61-64 et 109-111.

+ «Méditerranéennes 1», «Méditerranean Women 1», «Méditerranéennes 2», «Méditerranean Women 2», «Mon cœur amphithéâtre», «My Amphitheatre Heart», «Fille-mère», «Young Mother», "Anniversaire», «Anniversary», Henri-Dominique Paratte, (dir.), Poésie acadienne contemporaine/Acadian Poetry Now, Moncton, Éditions Perce-Neige, 1985, p. 73-85.

+ «Sérigraphie springtime», «Springtime No 2», «Springtime Nos 3 et 4 », «Poèmes pour les vieux couples», «Méditerranéennes 1», «Méditerranéennes 2», «Méditerranéennes 3», «Méditerranéennes 4 », «Impromptu», «Les cœurs longs», «Nos forêts exponentielles», «La racine du pain», Claude Beausoleil et Gérald Leblanc (dir.), La poésie acadienne 1948-1988, Trois-Rivières/Paris, Écrits des Forges/Le Castor Astral, 1988, p. 104109.

+ «Et cela dura», «Méditerranéennes 1», «Méditerranéennes 2», «Méditerranéennes 3», "Méditerranéennes 4 », Fred Cogswell et Jo-Ann Elder (dir), Rêves inachevés. Anthologie de poésie acadienne contemporaine, Moncton, Éditions d'Acadie, 1990, p. 66-70. Ces poèmes ont été publiés en traduction dans Fred Cogswell et Jo-Ann Elder (dir.), Unfinished Dreams. Contemporary Poetry of Acadie, Fredericton, Goose Lane Editions, 1990, p. 60-63.

+ «Poème impossible à finir», «Sur les traces de Marianne Godbout, cordonnière et savetière», «Comme Cavafy», «Le silence du fruit», «Mais où commence le corps?», Claude Beausoleil et Gérald Leblanc (dir.), La poésie acadienne, Moncton/Trois-Rivières, Éditions Perce-Neige/Écrits des Forges, 1999, p. 118-124. 


\section{5. In édits}

\section{5.1. Théâtre}

+ Moncton-Sable, Moncton, Moncton-Sable, 1997.

+ Le musée du nouvel âge, Moncton, Département de thêâtre de l'Université de Moncton, 1999.

+ Craie, Moncton, Moncton-Sable, 1999.

+ Foin, Moncton, Moncton-Sable, 2000.

+ Bric-à-brac, Moncton, Moncton-Sable, 2001.

\section{TRADUCTION DES ROMANS DE FRANCE DAIGLE}

+ Real Life: A Novel, traduction de Sally Ross, Don Mills (Ontario), Anansi, 1995, 83 p.

+ 1953: Chronicle of a Birth Foretold, traduction de Robert Majzels, Concord (Ontario), Anansi, 1997, 164 p.

+ Just Fine, traduction de Robert Majzels, Toronto, House of Anansi Press, 1999, 148 p.

+ A Fine Passage, traduction de Robert Majzels, Toronto, House of Anansi Press, 2002, 113 p.

\section{PRIX ET D ISTINCTIONS}

+ Prix Pascal-Poirier, en 1991, pour l'excellence en littérature francophone.

+ Prix Éloizes, en 1998, pour Pas pire.

+ Prix France-Acadie en 1998, pour Pas pire.

+ Prix Antonine-Maillet-Acadie vie, en 1999, pour Pas pire.

+ Prix Éloizes, en 2002, pour Pas pire.

\section{RÉCEPTION CRITIQUE ET ÉTUDES}

IV.1. Articles de fond et chapitres de livres

+ DÉLÉAS-MATTHEWS, Josette, «France Daigle: une écriture de l'exil, une écriture en exil», Atlantis, vol. 14, $\mathrm{n}^{\circ} 1,1988$, p. 122-126.

+ PLANTIER, René, «Sans jamais parler du vent de France Daigle: une écriture odyssée», Melvin Gallant (dir.), Mer et littérature. Actes du colloque international sur la mer dans les littératures d'expression française du vingtième siècle, Moncton, Éditions d'Acadie, 1992, p. 11-16.

+ OUELLET, Lise, «De l'autobiographie à la fiction autobiographique dans la littérature féminine», La Licorne, no 27, 1993, p. 365-378.

+ COOK, Margaret, «France Daigle: dualité et opposition», LittéRéalité, vol. 5, n 2, 1993-1994, p. 37-46.

+ PARÉ, François, «Cinémas. France Daigle», Théories de la fragilité, Ottawa, Le Nordir, 1994, p. 100-103.

+ PLANTIER, René, «La beauté de l'affaire: l'humour à plusieurs fils», Revue de l'Université de Moncton, vol. 27, nº 1, 1994, p. 161-176.

+ BOUDREAU, Raoul, «Le silence et la parole chez France Daigle», Raoul Boudreau, Anne Marie Robichaud, Zénon Chiasson et Pierre M. Gérin (dir.), Mélanges Marguerite Maillet, Moncton, Éditions d'Acadie, 1996, p. 71-81.

+ PLANTIER, René, Le corps du déduit. Neuf études sur la poésie acadienne 1980-1990: Huguette Légaré, Dyane Léger, France Daigle, Moncton, Éditions d'Acadie, 1996, p. 100-151.

+ — - «L'aléatoire dans l'excès des signes de la rigueur: La vraie vie de France Daigle», Raoul Boudreau, Anne Marie Robichaud, Zénon Chiasson et Pierre M. Gérin (dir.), Mélanges Marguerite Maillet, Moncton, Éditions d'Acadie, 1996, p. 313-324.

+ PARÉ, François, «La chatte et la toupie: écriture féminine et communauté en Acadie», Francophonies d'Amérique, no 7, 1997, p. 115-126.

+ RUNTE, Hans R., «Unwriting Writing», Writing Acadia: The Emergence of Acadian Literature 1970-1990, Amsterdam/Atlanta, Rodopi, coll. "Chiasma», nº 6, 1997, p. 153-163.

+ BOUDREAU, Raoul et Anne Marie ROBICHAUD, «Symétries et réflexivité dans la trilogie de France Daigle», Dalhousie French Studies, n 15, automne-hiver 1998, p. 143-153. 
+ GAUDET, Jeannette, «La métaphore du cinéma dans Film d'amour et de dépendance. Chef d'œuvre-obscur de France Daigle», Dalhousie French Studies, n 15, automne-hiver 1998, p.154-159.

+ MASSON, Alain, «Écrire, habiter», Tangence, n 58, 1998, p. 35-46.

+ PLANTIER, René, «Le renvoi de la balle acadienne: 1953 de France Daigle», Tangence, n 58, 1998, p. 56-65.

+ BOUDREAU, Raoul, «Les français dans Pas pire de France Daigle», Robert Viau (dir.), La création littéraire dans le contexte de l'exiguïté, Beauport, Publications MNH, coll. «Écrits de la francité», 2000, p. 51-64.

+ KELLETT-BETSOS, Kathleen L., «Histoire et quête identitaire dans 1953: chronique d'une naissance annoncée de France Daigle», Louis Bélanger (dir.), Métamorphoses et avatars littéraires dans la francophonie canadienne, Vanier, Éditions l'Interligne, 2000, p. 35-47.

+ ROY, Véronique, «La figure d'écrivain dans l'œuvre de France Daigle, aux confins du mythe et de l'écriture», Robert Viau (dir.), La création littéraire dans le contexte de l'exiguïté, Beauport, Publications MNH, coll. «Écrits de la francité», 2000, p. 27-50.

+ RICOUART, Janine, «France Daigle's Postmodern Acadian Voice in the Context of Franco-Canadian Lesbian Voices", Paula Ruth Gilbert et Roseanna L. Dufault (dir.), Doing Gender: Franco-Canadian Women Writers of the 1990s, Cranbury, Associated University Presses, 2001, p. 248-266.

+ ROY, Nathalie, «Le religieux dans Les fêtes de l'infini de J.R. Léveillé et La beauté de l'affaire de France Daigle: d'un emploi non référentiel des récits bibliques et préceptes chrétiens", Revue internationale d'études canadiennes, $\mathrm{n}^{\circ}$ 23, 2001, p. 37-55.

+ BOUDREAU, Raoul, "Choc des idiomes et déconstruction textuelle chez quelques auteurs acadiens», Robert Dion, Hans-Jürgen Lüsebrink et János Riesz (dir.), Écrire en langue étrangère: interférences de langues et de cultures dans le monde francophone, Québec, Nota bene, 2002, p. 287-304.

+ PALESHI, Stathoula, «Finir toujours par revenir: la résistance et l'acquiescement chez France Daigle», Francophonies d'Amérique, nº 13, 2002, p. 31-45.

+ DEN TOONDER, Jeanette, «Voyage et passage chez France Daigle», Dalhousie French Studies, n 62 , printemps 2003, p. 13-24.

+ FRANCIS, Cécilia W., "L'autofiction de France Daigle. Identité, perception visuelle et réinvention de soi», Voix et Images, no 84, printemps 2003, p. 114-138.

+ BOEHRINGER, Monika, «Une fiction autobiographique à plusieurs voix: 1953 de France Daigle», Revue de l'Université de Moncton, vol. 34, nos 1-2, 2003, p. 107-128.

+ GIROUX, François, "Sémiologie du personnage autofictif dans Pas pire de France Daigle», Francophonies d'Amérique, no 17, printemps 2004, p. 45-54.

+ DUMONTET, Danielle, «Le parcours autofictionnel de France Daigle entre opposition et résistance», Zeitschrift für Kanada-Studien, 24. Jahrgang, Nr. 1, Band 44, 2004, p. 86-100.

\section{V. 2. M é mo i r es}

+ LEPAGE, Renée, «Essai d'une poétique de l'interprétation: 1953: chronique d'une naissance annoncée de France Daigle», mémoire de maîtrise, Kingston, Université Queen's, 1997, 47 p.

+ PALESHI, Stathoula, «La constance des doubles chez France Daigle: finir par toujours revenir», mémoire de maîtrise, Waterloo, Université de Waterloo, 2001, 116 p.

+ WEISS, Véronique, «Les personnages et leur identité dans les romans de l'écrivaine acadienne France Daigle, La vraie vie, et de l'écrivaine québécoise Élise Turcotte, Le bruit des choses vivantes », Diplomarbeit, Autriche, Universität Klagenfurt, 2002.

\section{IV.3. Entretiens et portraits}

+ ROYER, Jean «Les chemins de l'Acadie mythique à l'Acadie réelle», Le Devoir, 15 décembre 1984, p. 30.

+ EL YAMANI, Myriame, «L'Acadie se comprend à mi-mots», Le Devoir, 10 octobre 1991, p. B6.

+ LAJOIE, Claudette, «Personnage remarqué», Femmes d'action, vol. 21, n² 2, 1991, p. 33-34. 
+ EL YAMANI, Myriame, «Elles réinventent l'Acadie», Châtelaine, août 1992, p. 78.

+ DÉSAUTELS, Sophie, «France Daigle. La Lelouch du roman», Ven'd'est, hiver 1993-1994, p. 54-55.

+ ALLARD, Claire, «La vraie vie de France Daigle: l'écriture», Atlantic Books Today, nº 6, été 1994, p. 3.

+ MARTEL, Réginald, «Folklore ou pas folklore», La Presse, 7 mai 1995, p. B1 et B4.

+ ARSENEAU, Marc, «France Daigle», Vallium, n 6, 1995, p. 38-42.

+ LEBLANC, Gérald, «France Daigle, le trésor bien caché», Le Journal, 26 avril 1997, p. 18.

+ MOUSSEAU, Sylvie, «L'agoraphobie au centre de Pas pire», L'Acadie nouvelle, 18 mars 1998, p. 25.

+ CAMPION, Blandine, «Éloge du plaisir et de la lenteur. France Daigle et l'espace cérébral», Le Devoir, 15 août 1998, p. D1-D2.

+ MOUSSEAU, Sylvie, «L'amour au quotidien de Terry et Carmen», L'Acadie nouvelle, 8 novembre 2002, p. 3.

+ SAINT-HILAIRE, Mélanie, «J'suis manière de proud de toi», L'actualité, vol. 27, n 3, 2002, p. 66-68.

+ BRUCE, Clint, «France Daigle et l'écriture au juste milieu», Le Front, 26 mars 2003, p. 16.

+ LEBLANC, Doris et Anne BROWN, «France Daigle: chantre de la modernité acadienne», Studies in Canadian Literature/Études en littérature canadienne, vol. 28, n 1, 2003, p. 147-161.

+ GIROUX, François, «Portrait d'auteure @ France Daigle», Francophonies d'Amérique, n 17, printemps 2004, p. $79-85$.

IV.4. Comptes rendus et articles de presse

IV.4.1. Sans jamais parler du vent. Roman de crainte et d'espoir que la mort arrive à temps

+ LANDRY, Bernadette, «Sans jamais parler du vent», Campus, 14e année, décembre 1983, p. 4.

+ + — «Sans jamais parler du vent: résumé», Dimensions, janvier 1984, p. 34.

+ BEAUSOLEIL, Claude, "Sans jamais parler du vent», Nuit blanche, n 12, février-mars 1984, p. 17.

+ BOUDREAU, Raoul, «Sans jamais parler du vent ou la Parole retenue», Le Papier, vol. 1, n 1, mars 1984, p. 14-15.

+ ALONZO, Anne-Marie, «D’Acadie: Sans jamais parler du vent», La Vie en rose, nº 19, septembre 1984, p. 56.

+ THALER, Danielle, "Sans jamais parler du vent. Roman de crainte et d'espoir que la mort arrive à temps", Resources for Feminist Research/Documentation sur la recherche féministe, vol. 14, nº 2, juillet 1985, p. 8.

\section{IV.4.2. Film d'amour et de dépendance. Chef-d'œuvre obscur}

+ ÉTIENNE, Gérard, «La saison acadienne s'ouvre magistralement», Le Devoir, 6 octobre 1984, p. 24.

+ ÉTIENNE, Natania, «La rentrée littéraire aux Éditions d'Acadie», Le P'tit Moniteur, 25 octobre 1984, p. 5.

+ LEBLANC, René, «France Daigle: Film d'amour et de dépendance», Le Courrier de la Nouvelle-Écosse, 21 novembre 1984, p. 12.

+ BEAUliEU, Michel, «Film d'amour et de dépendance», Livres d'ici, vol. 10, n 3, novembre 1984, p. 21-22.

+ OUELLET, Lise, «Film d'amour et de dépendance de France Daigle», Le Papier, n 3, novembre 1984, p. 18.

+ LÉPINE, Stéphane, «France Daigle, Film d'amour et de dépendance. Chef-d'œuvre obscur», Nos livres, vol. 15, n 5977, décembre 1984, p. 19-20.

+ ROSS, Sally, «Three Recent Acadian Literary Works», The Atlantic Provinces Book Review, vol. 12, nº 1 , février-mars 1985, p. 13.

+ TURCOTTE, Suzy, «Film d'amour et de dépendance», Nuit blanche, nº 17, février-mars 1985, p. 5.

+ ALONZO, Anne-Marie, "Chef-d’œuvre obscur», La Vie en rose, n² 24, mars 1985, p. 60-61.

+ MASSON, Alain, «France Daigle, Film d'amour et de dépendance», Revue de l'Université de Moncton, vol. 18, $\mathrm{n}^{\circ} 1,1985$, p. 162-164.

+ CORRIVEAU, Hugues, «France Daigle, Film d'amour et de dépendance (chef-d'œuvre obscur)», Estuaire, novembre 1986, p. 77-78. 
IV.4.3. Histoire de la maison qui brûle. Vaguement suivi d'un dernier regard sur la maison qui brûle

+ DE GONZAGUE, Louise, «Daigle (France), Histoire de la maison qui brûle. Vaguement suivi d'un dernier regard sur la maison qui brûle», Nos livres, vol. 17, nº 6613, juin-juillet 1986.

+ DORION, Hẻlène, «Histoire de la maison qui brûle», La Vie en rose, nº 40, novembre 1986, p. 62.

+ ROSS, Sally, «Acadian Publications», The Atlantic Provinces Book Review, vol. 13, n 4, novembre-décembre 1986, p. 14.

\section{IV.4.4. Variations en B et K. Plans, devis et contrat pour}

l'infrastructure d'un pont

+ DE GONZAGUE, Louise, «Daigle (France), Variations en B et K. Plans, devis et contrat pour l'infrastructure d'un pont», Nos livres, vol. 17, n ${ }^{\circ}$ 6497, mars 1986.

+ BROCHU, André, «Lascaux, les limbes et autres lieux», Voix et Images, n 34, automne 1986, p. 131-140.

\section{IV.4.5. L'été avant la mort}

+ LÉPINE, Stéphane, «D'un carnet intime et d'une conversation banale», Le Devoir, 2 août 1986, p. C2.

+ CÔTÉ, Suzanne, «L'été avant la mort», Canadian Woman Studies/Les Cahiers de la femme, vol. 7, n 3, automne 1986, p. 127.

+ ROSS, Sally, "Acadian Publications», The Atlantic Provinces Book Review, vol. 13, n 4, novembre-décembre 1986, p. 11-12.

+ MARCOTTE, Hélène, "L'été avant la mort», Québec français, nº 64, décembre 1986, p. 11-12.

+ POULIN, Gabrielle, «Prophétie et fiction. L'été avant la mort de France Daigle et Hélène Harbec», Lettres québécoises, n 43, 1986, p. 18-20.

\section{IV.4.6. La beauté de l'affaire. Fiction autobiographique à plusieurs voix sur son rapport tortueux au langage}

+ ROBICHAUD, Anne Marie, «La beauté de l'affaire de France Daigle», Éloizes, n 17, automne 1991, p. 81-84.

+ JACQUOT, Martine, «Jeux de mains, Mes simples et La beauté de l'affaire», Ven'd'est, no 46, novembredécembre 1991, p. 38.

+ COOK, Margaret, «La beauté de l'affaire de France Daigle», Francophonies d'Amérique, no 2, 1992, p. 63-64.

\section{IV.4.7. La vraie vie}

+ MARTEL, Réginald, «Une œuvre d’art, aussi riche que gratuite», La Presse, 12 septembre 1993, p. B5.

+ ALLARD, Jacques, «La vie qu'on raconte», Le Devoir, 18 septembre 1993, p. D5.

+ SERGENT, Julie, «La vraie vie», Voir, vol. 7, n 44, 30 septembre 1993, p. 32.

+ CRAN, E. Elisabeth, «La vraie vie: un roman absolument pas comme les autres», La Voix acadienne, 6 octobre 1993, p. 6.

+ MARTIN, Frédérick, «L’envers du décor et le décor du réel», Lettres québécoises, n 72, hiver 1993, p. 17-18.

+ BOUDREAU, Raoul, «La traversée du désir», Ven'd'est, n 58, hiver 1993-1994, p. 55.

+ BOUDREAU, Denis, «La vérité vraie du quotidien», Le Front, 2 février 1994, p. 14.

+ NICOL, Patrick, «La vraie vie», Mœbius, nº 61, 1994, p. 120-121.

\section{IV.4.8. 1953. Chronique d'une naissance annoncée}

+ CORRIVEAU, Hugues, «Effet de réel, effet de fiction», Trois, vol. 10, n 3, printemps-été 1995, p. 233-234.

+ GODIN, André, «1953», Le Front, 30 août 1995, p. 18.

+ ROSS, Sally, «Acadian Spring», Atlantic Book Today, n 10, été 1995, p. 8-9.

+ BORDELEAU, Francine, «L'écriture du roman», Lettres québécoises, n 79, automne 1995, p. 23. 
+ LEQUIN, Lucie, «Sur ma faim...», Voix et Images, n 62, hiver 1996, p. 387-389.

+ BÉLANGER, Georges, «1953. Chronique d'une naissance annoncée de France Daigle», Francophonies d'Amérique, nº 6, 1996, p. 39-41.

+ JACQUOT, Martine, «France Daigle. 1953. Chronique d'une naissance annoncée», LittéRéalité, vol. 8, nº 1, 1996, p. 144-145.

+ BOURQUE, Denis, «France Daigle. 1953. Chronique d'une naissance annoncée», Études francophones, vol. XII, no 1, printemps 1997, p. 192-195.

\section{IV.4.9. Pas pire}

+ GODIN, André, «Roman de l'essentiel ou simplement roman essentiel», Le Front, 8 avril 1998, p. 14.

+ LONERGAN, David, «Un beau et doux voyage», L'Accent acadien, vol. 3, n 48, 18 avril au 24 avril 1998, p. 6.

+ JACQUOT, Martine, "Pas pire, le nouveau roman de France Daigle», Le Courrier de la Nouvelle-Écosse, 24 avril 1998, p. 4.

+ CAMPION, Blandine, «Espace et écriture», Le Devoir, 2 mai 1998, p. D4.

+ MARTEL, Réginald, "Cohérence et densité sans faille», La Presse, 3 mai 1998, p. B2.

+ LAPLANTE, Laurent, "L'Acadie: mythes, défis et résonances», Nuit blanche, n 72, automne 1998, p. 56.

+ OLSCAMP, Marcel, «Regards acadiens sur l'institution littéraire», Spirale, nº 163, novembre-décembre 1998, p. 3.

+ MORENCY, Jean, «Pas pire», Éloizes, n 26, 1998, p. 155-157.

+ RICOUART, Janine, «Lectures acadiennes», Trois, vol. 15, nos 1-2-3, novembre 1999, p. 216-226.

+ CHASSAY, Jean-François, «Pas pire: roman de France Daigle», Francophonies d'Amérique, n 9, 1999, p. 5153.

\section{IV.4.10. Un fin passage}

+ MOUSSEAU, Sylvie, «Le casse-tête de France Daigle», L'Acadie nouvelle, 19 septembre 2001, p. 26.

+ GUY, Chantal, «Le monde à dos Daigle», La Presse, 23 septembre 2001, p. B3.

+ BOURGAULT-CÔTÉ, Guillaume, «Littérature de l'exil», Le Soleil, 29 septembre 2001, p. D10.

+ CHARTRAND, Robert, «De bien belles partances», Le Devoir, 29 septembre 2001, p. D3.

+ FORTIN, Marie-Claude, «Éloge de la fuite», Voir, vol. 15, n 40, 4 octobre 2001, p. 43.

+ LONERGAN, David, «Un fin et délicat passage vers la vie», L'Accent acadien, vol. 7, n²6, 30 novembre au 6 décembre 2001, p. 6.

+ MARCOTTE, Gilles, «L'amour existe-t-il encore?», L'actualité, vol. 26, n 19, $1^{\text {er }}$ décembre 2001, p. 95-96.

+ MONTESSUIT, Carmen, «France Daigle aime écrire par fragments», Le Journal de Montréal, 23 décembre 2001, p. 37.

+ JOUBERT, Lucie, «Des retours attendus», Spirale, n 187, novembre-décembre 2002, p. 54-55.

+ CORMIER, Pénélope, «Dans une semaine, la vie», Éloizes, n 31, 2002, p. 96-98.

\section{IV.4.11. Petites difficultés d'existence}

+ GIGUÈRE, Suzanne, «Il y a des lapses dans ce pays», La Presse, 6 octobre 2002, p. F2.

+ BIRON, Michel, «Les petites marées», Le Devoir, 16 novembre 2002, p. F3.

+ LONERGAN, David, «France Daigle: un univers de lumière», L'Acadie nouvelle, 29 novembre 2002, p. 6.

+ BIRON, Michel, «Le symbolisme soft», Voix et Images, n 83, hiver 2003, p. 167-173 [170-172].

+ LAURIN, Danielle, «France Daigle. L'Acadie aujourd'hui», Elle Québec, janvier 2003, p. 26. 